Research article

\title{
Novelties in the genus Cerastium (Caryophyllaceae) in Turkey
}

\author{
Mustafa Keskin $^{1}$ iD \\ ${ }^{1}$ Marmara University, Institute of Pure and Applied Sciences, Biology Program, 34722, Goztepe, Istanbul, Turkey
}

\begin{abstract}
The novelties of the genus Cerastium in Turkey were presented in the study along with the description of three new taxa: Cerastium comatum var. longipedicellatum, $C$. inflatum var. longum, and C. semidecandrum var. delicatum. So, two new combinations have been made for the first time. Distribution records of all mentioned taxa, along with photographs of the new taxa, were also provided.
\end{abstract}

Keywords: Caryophyllaceae; Cerastium; morphology; new taxa; new combinations; taxonomy; Turkey

\section{Introduction}

Caryophyllaceae Juss. is a large family, more frequent in the temperate regions of Northern Hemisphere (Bittrich 1993). The genus Cerastium L. belongs to the subfamily Alsinoideae Fenzl. This genus is represented with about 31 species (Cullen 1968; Davis 1988), and later Keskin (2012) reported 36 species from Turkey.

During a taxonomic revision of the genus Cerastium for New Illustrated Turkish Flora, three different taxa were seen in Herbarium of the Faculty of Pharmacy of Istanbul University (ISTE) and Ege University Faculty of Science Herbarium (EGE). The first two taxa are separated from the $C$. comatum $\mathrm{L}$. and $C$. inflatum Link ex Boiss. by their longer pedicels. The third taxon is isolated from $C$. semidecandrum $\mathrm{L}$. by the short stem, short and non-recurved pedicel, etc.

In this article, three new taxa (Cerastium comatum var. longipedisellatum, $C$. inflatum var. longum, and $C$. semidecandrum var. delicatum) were introduced to the world of science and two new combinations ( $C$. ligusticum var. dominicii and $C$. dichotomum var. saccardoanum) were made.

\section{Materials and methods}

The studied materials in ISTE and EGE herbaria were examined.

The vegetative and reproductive characteristics of specimens were checked by stereomicroscope. Samples were evaluated using several local, and neighboring Floras (Shishkin 1936; Jalas and Whitehead 1964; Cullen 1967; Strid (1997); Moschl 1988), also checked related articles (Grenier, 1841; Keskin and Cirpici 2006).

\section{Results}

\subsection{Cerastium comatum Desv. J. Bot. Agric. 3(5): 228 (1816)}

Annual, 1-12(-20) cm high. Stems more branched from the base and divaricately, covered retrorsely hairy. Nods longer than leaves. Leaves obovate-oblong to spathulate at base; others narrowly obovate to oblong, 5-10 x 2.5-4 mm, oblong-ovate, obovate, thin hairy, somewhat congested at the base. Inflorescences loose with a few flowers. Pedicels $2-13 \mathrm{~mm}$, shorter than sepals or rarely longer than sepal, hairy-like stem. Sepals 4-5 mm, linear-lanceolate, margin without scarious margin or very narrow; hairs longer than sepals. Petals 2.5-4 $\mathrm{mm}$, shorter than sepals, clav glabrous. Filaments glabrous at base. Capsule shorter than sepals, teeth very short. Seeds small and dark brown.

It is a very different taxon with long and mainly patent

\footnotetext{
* Corresponding author.

E-mail address: trifolium@ hotmail.com (M. Keskin).

https://doi.org/10.51753/flsrt.904030 Author contributions

Received 26 March 2021; Accepted 4 May 2021

Available online 7 May 2021

2718-062X (C) 2021 This is an open access article published by Dergipark under the CC BY license. 
calyx hairs and pored fruiting capsules due to very small teeth. This taxon, which little known species was introduced to the world of science with a short description (Cerastium comatum, Nob. Annuuin, pilosum: caule erecto aut diffuso, piloso: pilis reflexis; foliis ob-ovato-spathulatis; calice piloso-crinito: pilis sub-in-flexis. Habitat in Oriente.) by Desvaux (1814).

It is related to the $C$. illyricum but is easily distinguished by long sepals with wide scarious margin and its hairs not exceeding apex; petals are nearly equaling sepals.

\subsection{1. var. comatum (Fig. 1. A and B)}

Stems more branched from the base and divaricately. Pedicels 2-10 $\mathrm{mm}$, shorter than sepals or rarely longer than sepal. Sepals 4-5 mm, linear-lanceolate, without scarious margin or very narrow. Capsule shorter than sepals.

\section{Examined materials in Turkey:}

Balıkesir: Ayvalık, South of Güneş Island, Yumurta island, 16.iv.1998, 5 m, K.Alpınar (ISTE 75011!). Marmara Islands, east of center, -hills above lime quarries- 8.v.1971, A.Baytop, G.Ertem, F.Öktem (ISTE 19633!). Marmara Island, Tops the road from the village of Palaces to the top of the Radar, 1.v.1978, 360 m, E.Tuzlacı (ISTE 39263!). Denizli: Babadağ, near to Altındere Village, 3.vi.1980, 800 m, E.Tuzlac1, T.Çelebioğlu (ISTE 44509!). İstanbul: Adalar, Burgazada, iv.1910, S.Selinka (ISTF 15!). Sarıyer, Rumeli Feneri, Kale District, 29.iv.2003, M. Keskin 2623!. İzmir: Dilek Peninsula, 1500 m, 25.v.1995, T.Ekim (GAZI 9437!). Manisa Mount, 13.vi.1986, c.1500 m, A.Baytop, B.Çubukçu, T.Avcıgil (ISTE 9983!). Ödemiş, Keldağ -forest openings-, 31.v.1980, 1200m, E.Tuzlacı, T.Çelebioğlu (ISTE 44370!). Selçuk Way, $10 \mathrm{~km}$ to Selçuk, 9.iv.1971, A. and T. Baytop (ISTE 19089!). Muğla: Ortaca, Dalyan, Arıktaş Mah. Göktepe, shrubs, limestone land, 19.iv.1991, H.Duman, A.Dönmez, B.Mutlu (GAZI!). Tekirdağ: İnecik-7.2 km before Yenice, 18.iv.1992, A.Byfield 15 (ISTE 64841!). Seymen to Çorlu, 22.iv.1970, A.Baytop, F.Öktem (ISTE 17601!).

\subsection{2. var. longipedicellatum M.Keskin var. nov. (Fig. 1. C} and $D)$

Diagnose: It is easily distinguished from the main taxon by its very long pedicels (alar fruiting pedicels $25-35 \mathrm{~mm}$; other pedicels $8-25 \mathrm{~mm}$ ).

Description: Annual, 9-13 cm high. Stems single, short and recurved hairy. Leaves spathulate $9-10 \mathrm{~mm}$, obtuse at the apex, and short-hairy. Upper leaves oblong-lanceolate, long hairy. Alar fruiting pedicels $25-35 \mathrm{~mm}$, ascending up. Sepals 5$6 \mathrm{~mm}$, acute, medium scarious at the margin. Other pedicels 8$25 \mathrm{~mm}$. Sepals patent hairy as wide of the sepal. Petals 3-3.5 $\mathrm{mm}$, glabrous. Style 0.7-0.8 mm. Stamens as long as ovarium; filaments glabrous at base. Capsule equal to subexerted from the calyx.

Holotypus: Türkiye, Balıkesir, Ayvalık, Maden Adası, 29.iv.1997, 30 m, K. Alpınar ISTE 74000!.

\subsection{Cerastium inflatum Link ex Boiss., Fl. Orient. 1: 721 (1867)}

Annual herbs. Stems $5-15 \mathrm{~cm}$, strict, simple or branched at base; glandular and simple hairy but abundant glandular.
Leaves, pilose at the edge, narrowly ovate to lanceolate, acute at tip; amplexicaul at the middle part. Inflorescences strict, a few flowers. Bracts leaf-like, tiny pilose. Sepal ovate 10-12 mm, widened at the fruiting time; glandular-hairy; membranous at the edge. Petal 8-12 mm, as long as sepal, emarginate at the tip. Stamens 10, filaments hairy at base. Stillus 5. Capsule strict, conical, and longer than sepal. Seeds 0.7-0.9 mm, brownish, tuberculate.

\subsection{1. var. inflatum (Fig. 2. A and B)}

Stems 10-15 mm. Leaves 10-30 x 2-5 mm. Pedicels shorter than sepal and strict.

\section{Examined materials in Turkey:}

Adana: Karsant1, Torosan Mountain, Kanliyalak area, 2160 m, 15.vi.1977, E.Yurdakulol (ANK 1098!). Seyhan, Bozoğlan Mount, 2000 m, Davis 19727 and R.Çetik (ANK!). Adıyaman: Gölbaşı, around the village of Yukçöplu, Limestone, vineyard, $37^{\circ} 45^{\prime} 843^{\prime}$ ' $\mathrm{K}$, 37 $37^{\circ} 43^{\prime} 039^{\prime}$ ', $1000 \mathrm{~m}$, 18.iv.2001, A.A.Dönmez 8572 (HUB!).Antalya: Elmalı, Çığlıkara, Kabakçılar area, Alpin, 2100 m, 27.vi.1975, R.Çetik (ANK 1586!). Diyarbakır: Merkez, 2 km w. Ambar Stream, field edge, 635 m, 6.v.1976, H.Misirdalı and H.Malyer (ISTF 29411!). Elazığ: Keban, 1000-1200 m, 11.vi.1983, T.Ekim (ANK 7159!). Erzincan: Kemaliye, Water mains start, Limestone, Quercus-Paliurus opening, 16.iv.2001, A.A.Dönmez 8563 (HUB!). Kahramanmaraş: Ahırdağ1, around the transmitter station, Ulucak Hill, Bakacak view, oak openings, 1200-1500 m, 21.v.1992, Z.Aytaç et H.Duman (ANK 4596!). Engizek Mountain, Aksu District area, field edge, 10001200 m, 24.v.1987, H.Duman 1988 (ANK 2922!). GöksunKahramanmaraş, Tekne Mountain, 1600 m, 4.v.1957, Davis 27548 et Hedge (E!). Konya: Ermenek, Damlaçal, Juniperus excelsa, stony places, $1700 \mathrm{~m}, 27 . \mathrm{v} .1979$, M.Vural (ANK 1666!). Hadim, Taşkent, Ermenek Road, Feslikan Plateau, limestone rock, 1600-1800 m, 16.v.1984, S.Erik 3634, B.Y1ldız (HUB 02754!). Malatya: Malatya-Akçadağ, $1000 \mathrm{~m}$, 10.v.1957, Davis 27689 et Hedge (ANK! and E!). MalatyaKayseri road, $36 \mathrm{~km}$ from Malatya, 9.vi.1971, A.Baytop (ISTE 20179!). Mardin: Mardin-Diyarbakır, 1000 m, 27.v.1957, Davis 28725 et Hedge (ANK! and E!). Mardin: Merkez, 1100 m, 21.v.1957, Davis 28392 et Hedge (ANK!). Mardin: Merkez, steep, limestone vineyards, 1100 m, 21.v.1957, Davis 28392 et Hedge (E!). Savur to Midyat, $13 \mathrm{~km}$ from Savur, $100 \mathrm{~m}$, 5.v.1966, Davis 42497 (E!). Savur, vi.1888, P.Sintenis 871 (E!). Mersin: Mut, Mağras Mountain, summit, limestone rocks and deep pine needle, $1400 \mathrm{~m}$, 11.v.1965, M.J.E.Coode and B.M.G.Jones (E!). Muş: Malazgirt, Aktuzla, Kocasuyu Edge, step, 1550 m, 12.v.2001, S.Almanar 1336 (VANF 3668!). Siirt: above Şırnak, rocky limestone slopes, 1400-1500 m, 8.v.1966, Davis 42593 (E!). Sivas: Divriği, 31.v.1968, T.Baytop (ISTE 12942!). Sivas: Divriği, Dumluca mountain, 30.v.1968, T.Baytop (ISTE 12912! and E!). Van: Erciş, Yukarı Işıklı

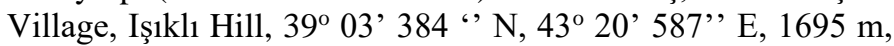
15.v.2006, O.Karabacak (VANF 3673!). Van: Gürpınar, Bölmeçalı Village around, step, 2000 m, 10.vii.2002, M.Ünal 7293 (VANF 3670!). Van: Gürpınar, Hamurkesen Village, step, 2000 m, 22.v.2002, M.Ünal 6749 (VANF 3669!). Gürpınar, Zernek Dam and Üçgen Village, stream edge, 1700-1900 m, 10.vi.2007, İ.Demir 432 (VANF 3691!). 


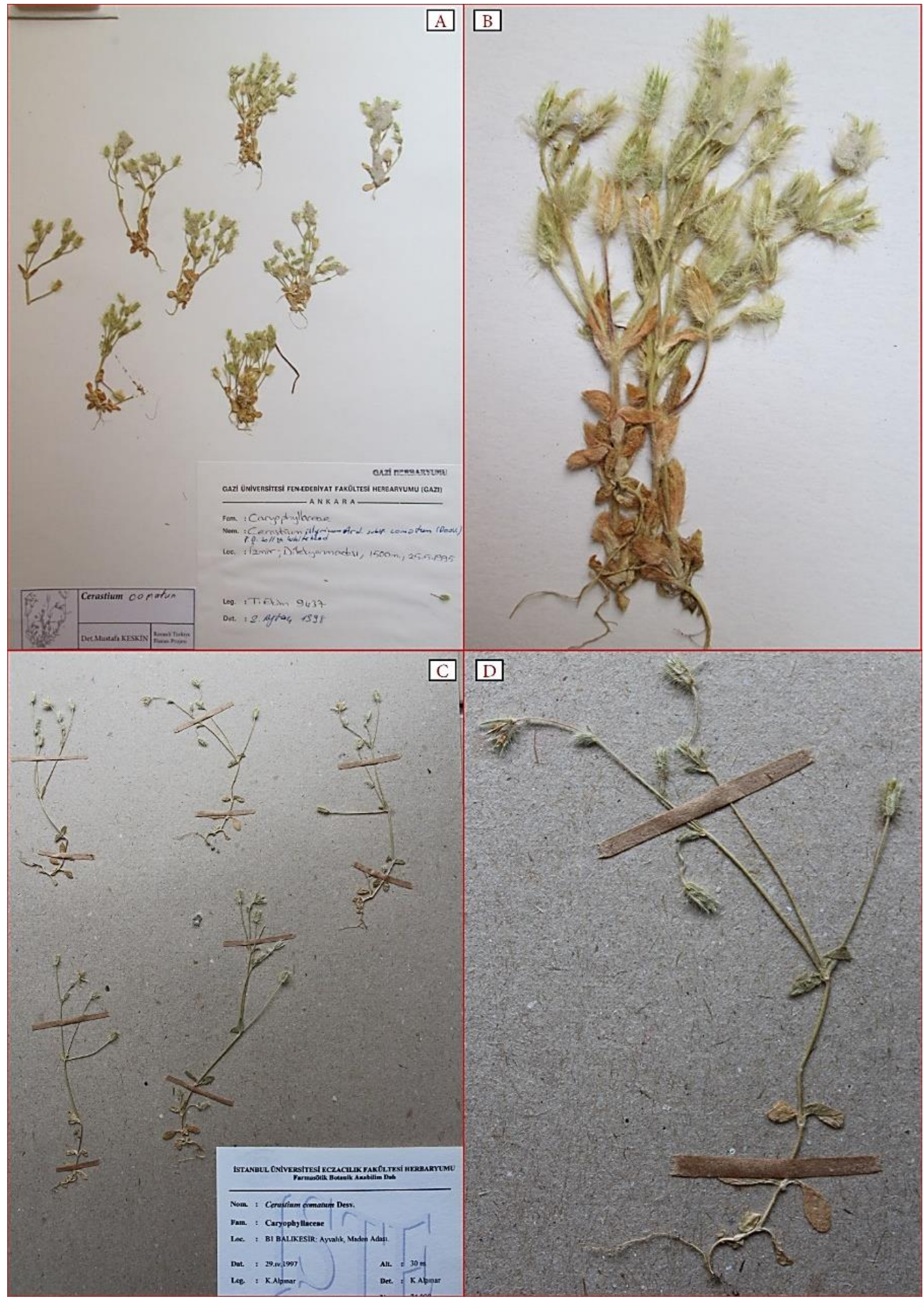

Fig. 1. (A) Cerastium comatum var. comatum (a herbarium sheet), (B) C. comatum var. comatum (single plant), (C) C. comatum var. longipedicellatum (a herbarium sheet), (D) C. comatum var. longipedicellatum (single plant). 


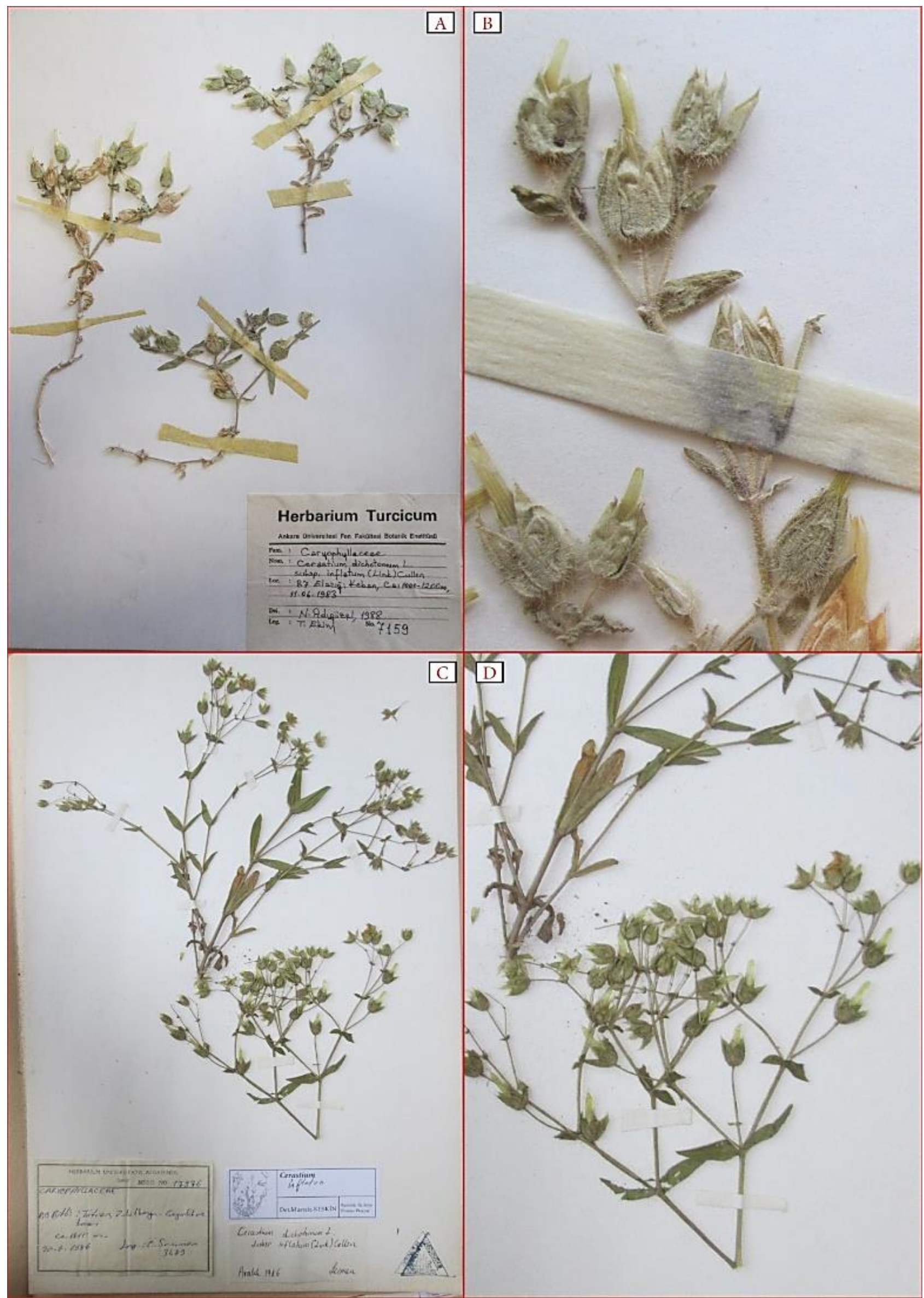

Fig. 2. (A) Cerastium inflatum var. inflatum (a herbarium sheet), (B) C. inflatum var. inflatum (close up) (C), C. inflatum var. longum (a herbarium sheet) (D), C. inflatum var. longum (single plant). 


\subsection{2. var. longum M.Keskin var. nov. (Fig. 2. C and D)}

Diagnose: It is distinguished from the main taxon by its long pedicels, leaves, and stem.

Description: Stem probably 20-40 cm. Leaves 40-55 x 9$13 \mathrm{~mm}$, wide oblong to lanceolate, obviously obtuse at apex. Inflorescences more flowered. Alar pedicels 40-45 mm. Other pedicels $15-30 \mathrm{~mm}$. Bracts leaf-like but very small. Sepals 9-12 $\mathrm{mm}$, narrowly membranous at the edge. Petal shorter than sepal Capsule 20-25 mm, slightly conical.

Holotypus: Türkiye, Bitlis, Tatvan, Zulül kaya, çayırlık ve kenar1, ca.1850 m, 20.vi.1986, Ö.Seçmen 3489 (EGE 17976!).

\subsection{Cerastium semidecandrum L., Sp. Pl. 1: 438 (1753)}

Annuals. Stem 1-20 cm, thinly; spreading 2-3 celled eglandular with a few minute glandular hairs present, especially at the leaf base. Leaves spathulate at the base, others oblong; hairy on the upper face, glabrous or loosely hairy on the lower face. Inflorescences usually single-flowered. Bracts membranous at tips, sometimes only greenish in the middle, other parts membranous. Pedicels usually equal to sepal or longer than sepals. Sepals ovate-lanceolate, narrowly membranous at the edge and widely membranous and purplish at tip; dense and long hairs with 2-3 cell on edge: usually in a line or very sparsely hairy or absent in the middle; no glandular hairs or few tiny glandular hairs near the pedicel (these are very small and can be seen at high magnification). Petals shorter than sepals, nearly as long as middle part; glabrous, entire or 2-3 irregular parted and sometimes purplish at tip; glabrous. Filaments as long as petals; anthers sphere-like. Style $0.5 \mathrm{~mm}$. Capsule 2-7 mm. Seeds 0.4$0.8 \mathrm{~mm}$, bluntly tuberculate.

\subsection{1. var. semidecandrum (Fig. 3. A and B)}

\section{Examined materials in Turkey:}

Antalya: Kemer, Kardalan-Kızder Village, Pinus brutia openings, 100-1000 m, 7.vi.1979, H.Peşmen 4366 and A.Güner (HUB 02843!). Bilecik: Söğüt-Eskişehir $11 \mathrm{~km}$, before $1 \mathrm{~km}$ from Gündüzbey, roadside, $1020 \mathrm{~m}$, 13.vi.1954, H.Demiriz (ISTF 13239!). Söğüt-Eskişehir, 24 km, 2 km after Oluklu, step, hill, 13.vi.1954, H.Demiriz (ISTF 13260!). Bursa: above Çekirge, roadside, 19.iv.1952, A.Berk and T.Baytop (ISTE 2531!). Edirne: Lalapaşa, Süloğlu Village way, 30.iv.1973, G.Ertem (ISTE 24267!). Eskişehir: Sündiken Mountains, Kirazlı Stream, Marg1 area, Meadows, 1250 m, 24.iii.2006, A.A.Dönmez 12711 and A.Ocak (HUB!). İstanbul: Adalar, Burgazada, 13.v.2004, M.Keskin 3257!. Adalar, Heybeliada, 13.v.2004, M.Keskin 3250!. Adalar, Kınalıada, 13.v.2004, M.Keskin 3278!. Bahçelievler, Park, 12.iv.2004, M.Keskin 3105!. Bakırköy, Yeşilköy, around siteler, 12.iv.2004, M.Keskin 3098!. Büyükçekmece, lake area, 17.iv.2004, M.Keskin 3111!. Çatalca, Çatalca mountain summit, 17.iv.2004, M.Keskin 3107!. Eminönü, Ayasofya Mosque, tea garden perimeter, 12.iv. 2004, M.Keskin 3092!. Eminönü, Sirkeci train station perimeter, 12.iv.2004, M.Keskin 3090!. Küçükçekmece, lake road, roadside, 17.iv.2004, M.Keskin 3114!. Maltepe, Başıüyük District, Süreyyapaşa Hospital forest, near the pond, 25.iv.2004, M.Keskin 3115!. Sarıyer, Rumeli Feneri, Kale District, 29.iv.2003, M.Keskin 2619!. Tuzla, 29.iv.1962, B.Tözün (ISTF 18366!). Ümraniye, Alemdar, Nişan Hill forest openings, 24.iv.2004, M.Keskin 3127!. Kırklareli: Lüleburgaz, Pınarhisar-Lüleburgaz $16 \mathrm{~km}$, field edge, meadow, 5.v.1974, B.Tütel (ISTF 27685!). Kütahya: Gediz, 21 km, Göksuyu right bank, lime rocks, 960 m, 14.vi.1954, H.Demiriz (ISTF 13303!).

Tekirdağ: Naipköy-Işıklar road, Bin Hill, 30.iv.1967, A.Baytop and T.Baytop (ISTE 10853a!).

\subsection{2. var. delicatum M.Keskin var. nov. (Fig. 3. D and E)}

Diagnose: Allied to $C$. semidecandrum L. but easily distinguished by single-flowered inflorescences; short and nonrecurved pedicels; short calyx and capsules; eglandular indumentum.

Description: Stem 1-2.5 cm; spreading 2-3 celled eglandular with a few minute glandular hairs present, especially at the leaf base. Leaves 1-7 $\mathrm{mm}$, spathulate at the base. Inflorescences usually single-flowered. Sepals 1-3 mm, narrowly membranous at the edge and widely membranous and purplish at the tip (1/3-1/4); dense and long hairs with 2-3 cell on edge. Capsule $2 \mathrm{~mm}$.

Holotypus: Turkey, İzmir: Bergama-Kozak, çayırlık, 400 m, 27.ii.1986, G.Görk (EGE 315601!).

\subsection{New Combinations}

\subsubsection{Cerastium ligusticum Viv. (Fig. 4. A and B)}

The species is growing in a narrow area in Turkey. Its long petals are the most prominent characteristic of this species. $C$. dominicii Favarger was described from Muğla in 1976. Later, it was validated by Tan and Mill in 1988. Both species grow around Muğla. According to my observations, the only difference from C. ligusticum is its large seeds.

C. ligusticum Viv. var. dominicii (Kit Tan and R.R.Mill) M.Keskin. comb. nov. (Fig. 4. C and D)

\subsubsection{Cerastium dichotomum L. (Fig. 5. A and B)}

This species is common in mainly Central Anatolia. The stem of this species is fork-like branched, and extended conical capsules are quite typical. C. saccardoanum Diratz. is published in 1912. This species differs with its capsules as long as the sepal. But this character alone is not enough for the species level.

C. dichotomum L. var. saccardoanum (Diratz.) M.Keskin comb. nov. (Fig. 5. C and D)

\section{Conclusion}

In this article, some observations during the revision of Cerastium distributed in Turkey are reported. During the revision study, three interesting taxa were encountered in ISTE and EGE herbarium.

As a result of examining these samples, it was decided that these are new taxa for science. These examples are introduced to the scientific world in this article: $C$. comatum Desv. var. longipedicellatum M.Keskin, C. inflatum Link ex Boiss. var. longum M.Keskin and C. semidecandrum L. var. delicatum M.Keskin.

Also, two new combinations have been made: $C$. ligusticum Viv. var. dominicii (Kit Tan and R.R.Mill) M.Keskin and C. dichotomum L. var. saccardoanum (Diratz.) M.Keskin. 


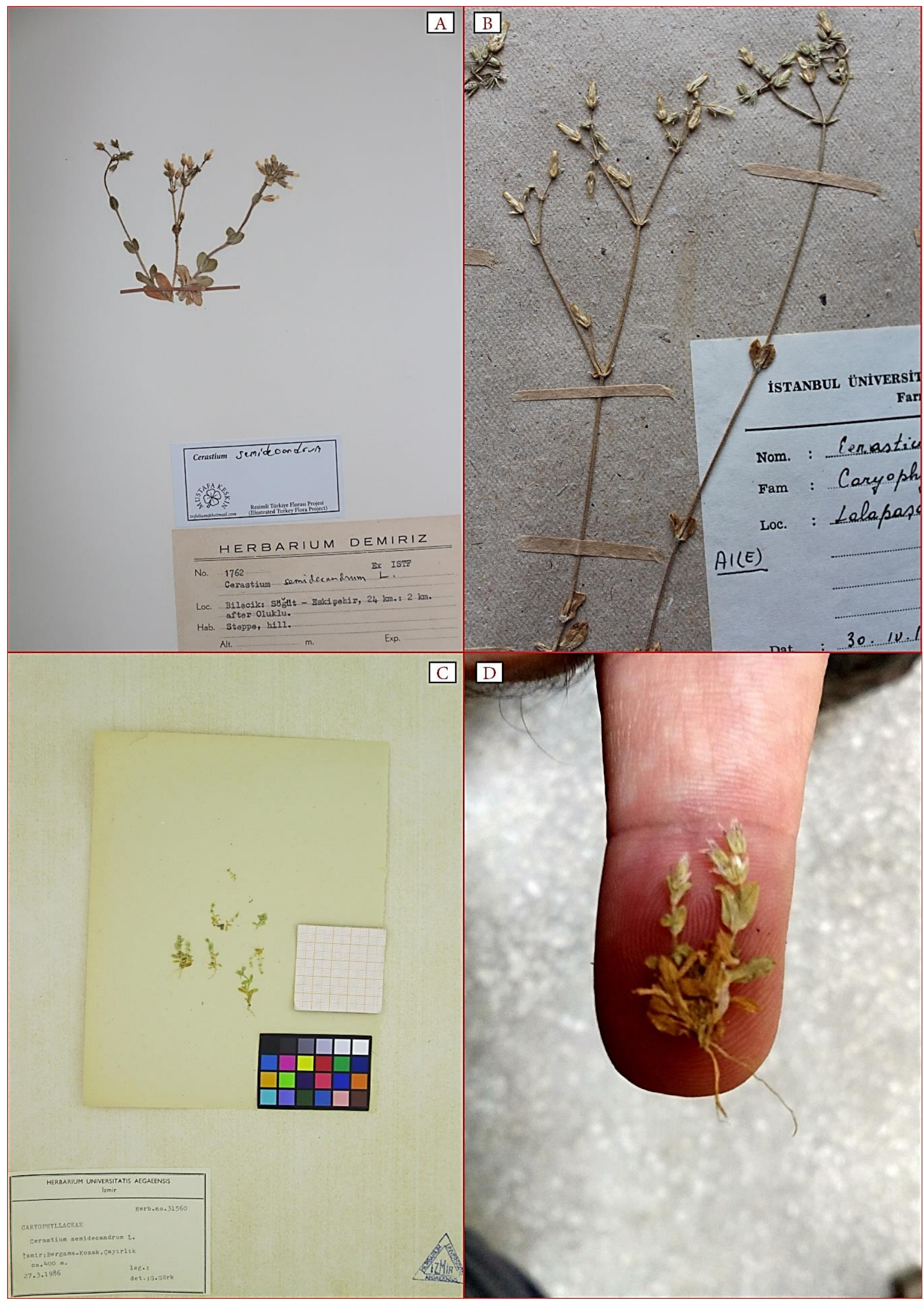

Fig. 3. (A) Cerastium semidecandrum var. semidecandrum (a herbarium sheet), (B) C. semidecandrum var. semidecandrum (single plant), (C) C. semidecandrum var. delicatum (a herbarium sheet), (D) C. semidecandrum var. delicatum (single plant). 


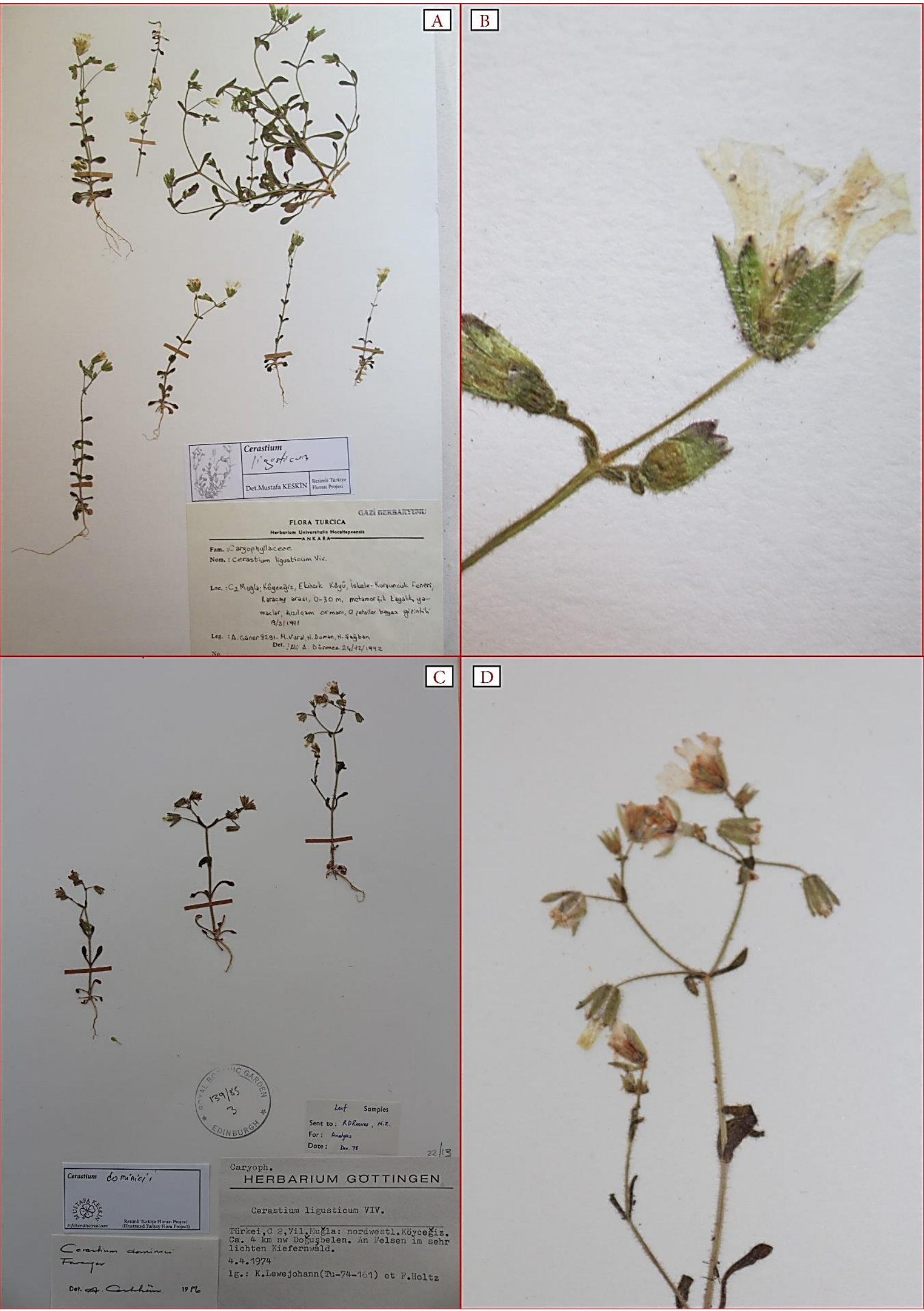

Fig. 4. (A) Cerastium ligusticum var. ligusticum (a herbarium sheet), (B) C. ligusticum var. ligusticum (close up), (C) C. ligusticum var. dominicii (a herbarium sheet), D) C. ligusticum var. dominicii (single plant). 


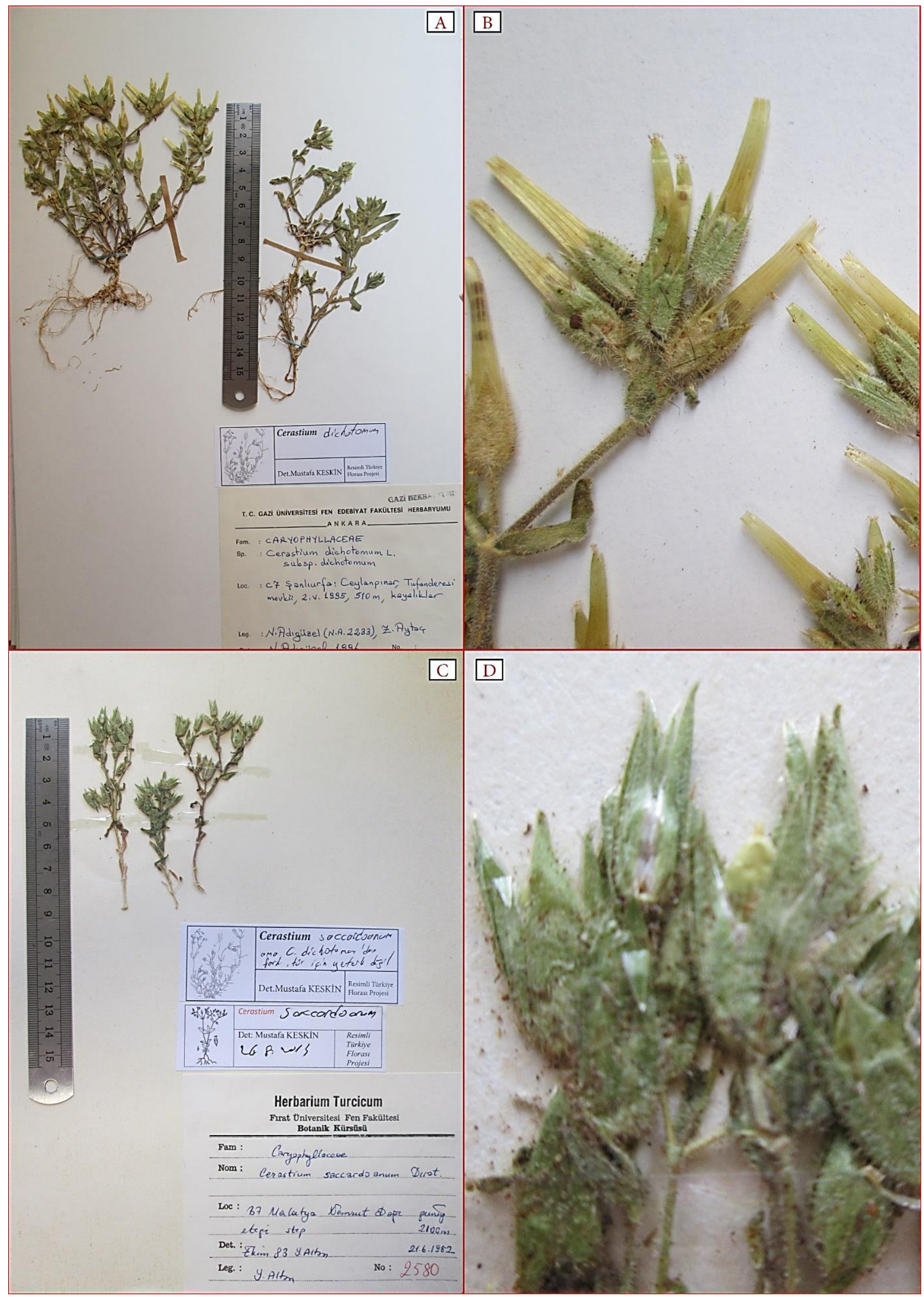

Fig. 5. (A) Cerastium dichotomum var. dichotomum (a herbarium sheet), (B). C. dichotomum var. dichotomum (close-up), (C) C. dichotomum var. saccardoanum (a herbarium sheet), (D) C. dichotomum var. saccardoanum (close-up). 
Conflict of interest: The author declares that he has no conflict of interests.

\section{References}

Bittrich, V. (1993). Caryophyllaceae. In: Kubitzki K. (eds) The Families and Genera of Vascular Plants 2 (pp. 206-236). Springer-Verlag, Berlin.

Cullen, J. (1967). Cerastium L. In: Davis P. H. (ed) Flora of Turkey and the East Aegean Islands 2 (pp. 73-85). Edinburgh at the University Press.

Davis, P. H. (1988). Flora of Turkey and the East Aegean Islands vol. 10 (pp. 69-71). Edinburgh at the University Press.

Desvaux, N. A. (1814). Examen Critique de Genres Saline (Arenaria) et (Cerastium). Journal de Botanique Appliquee A L'Agriculture, A La Pharmacie, A La Médecine et Aux Arts. Paris. No: III, Ier, vol. 3: 213 228.

Grenier, C. (1841). Monographia de Cerastio: Cum tab. 9 vol. 1 (pp. 1-85). Outhenin-Cahlandre.

Jalas, J., Sell, P. D., \& Whitehead, F. H. (1964). Cerastium L. In: Tutin T. G., Heywood V. H., Burges N. A., Valentine D. H., Walters S. M.,
Informed consent: This manuscript did not involve human or animal participants; thus informed consent was not collected.

Webb D. A. (eds) Flora Europaea 1 (pp. 136-145). Cambridge at the University Press.

Keskin, M. (2012). Cerastium L. In: Güner A., Aslan S., Ekim T., Vural M., Babaç M. T. (eds) Türkiye Bitkileri Listesi (Damarlı Bitkiler) (pp. 329333). Nezahat Gökyiğit Bahçesi ve Flora Araştırmaları Derneği Yayını. İstanbul.

Keskin, M., \& Cirpici, A. (2006). İstanbul çevresinin Cerastium L. (Caryophyllaceae) türleri üzerinde taksonomik araştırmalar. OT Sistematik Botanik Dergisi, 13(1), 71-96.

Moschl, W. (1988). Cerastium L. In: Rechinger K. H. (ed) Flora Iranica 163 (pp. 85-108). Akademische Druck-u. Verlagsanstalt, Graz, Austria. Shishkin, B. K. (1936). Cerastium L. In: Komarov V. L. (ed) Flora of the U. S. S. R. 6 (pp.430- 466). Leningrad.

Strid, A. (1997). Cerastium L. In: Strid A., Tan K. (eds) Flora Hellenica vol.1 (pp. 198-214). Koeltz Scientific Books, Germany.

Cite as: Keskin, M. (2021). Novelties in the genus Cerastium (Caryophyllaceae) in Turkey. Front Life Sci RT, 2(2), 35-43. 